\title{
Interpreting Nestedness and Modularity Structures in Affiliation Networks: An Application in Knowledge Networks Formed by Software Project Teams
}

\author{
Jorge Luiz dos Santos ${ }^{1}$, Renelson Ribeiro Sampaio ${ }^{2}$ \\ ${ }^{1}$ Federal Data Processing Service-Serpro, Salvador, Brazil \\ ${ }^{2}$ University Center, Senai Cimatec, Salvador, Brazil \\ Email: jorgeluizdossantos@gmail.com, renelson@fieb.org.br
}

How to cite this paper: dos Santos, J.L. and Sampaio, R.R. (2021) Interpreting Nestedness and Modularity Structures in Affiliation Networks: An Application in Knowledge Networks Formed by Software Project Teams. Social Networking, 10, 1-18. https://doi.org/10.4236/sn.2021.101001

Received: October 10, 2020

Accepted: January 9, 2021

Published: January 12, 2021

Copyright $\odot 2021$ by author(s) and Scientific Research Publishing Inc. This work is licensed under the Creative Commons Attribution International License (CC BY 4.0).

http://creativecommons.org/licenses/by/4.0/

(c) (i) Open Access

\begin{abstract}
An understanding of the knowledge creation and diffusion process in the organizational context is extremely relevant. Because from this understanding, organizations can restructure processes, reorient teams and implement methodologies to assist in the construction of an evolutionary process of knowledge creation and diffusion aimed at sustainable growth and innovation. The theory of complex social networks has been applied in several fields to help understand organizational cognitive processes. However, these approaches still insipiently consider the analysis of the nestedness and modularity of the studied networks. In this article, we presented an approach that sought to identify patterns of nestedness and modularity in networks of affiliation of people in projects in the organizational context. The study sought to identify these patterns in affiliation networks in a public organization providing information technology services in the period from 2006 to 2013. The detection of these patterns was performed using the NODF (Nestedness metric based on Overlap and Decreasing Fill) algorithm described by [1]. The nestedness and modularity metrics can influence patterns of knowledge creation and diffusion in formal and informal networks constituted for the execution of projects in organizations. This study showed that the network structures of the organization during the study period presented a high degree of nestedness, and it was possible to identify combined structures of nestedness and modularity.
\end{abstract}

\section{Keywords}

Social Network Analysis, Affiliation Networks, Modularity, Nestedness 


\section{Introduction}

Modeling based on complex network analysis has been used as a tool to answer questions and identify characteristics, behaviors and cause-and-effect relationships involving the systems studied in many areas of knowledge. Some of these questions are not typically visible when studied from the perspective of other analytical approaches. An example of characteristics related to the interactions between the components of these networks that are not necessarily visible in the light of other approaches is the nestedness and modularity metrics, as described by [2]. The study of these measures has helped to understand network structures formed in biological systems and has also been applied to economic systems. In the field of biology, according to [2], the study of these characteristics helps to formulate important hypotheses. These hypotheses may, for example, lead to a better understanding of species behavior, interaction between species or interaction between species and the environment. In the field of economics, such studies were a complementary part of research that resulted in predictive models about economic development or about the emergence and disappearance of certain industries and companies in several countries, such as the studies conducted by [3] and also by [4].

In the area of administration encompassing the various branches of organizational management, such as people management, logistics management and project management, several applications based on the analysis of social and complex networks have already been the subject of research and case studies, as reported by [5]. However, these approaches still do not address the issue of nestedness and modularity in these networks in a more consistent manner. Therefore, conducting studies involving metrics of nestedness and modularity can also help elucidate important issues in the organizational context, since these metrics reflect forms of groupings and interactions that can occur among groups formed in organizations. In this case, one of the possible applications is the study of work activity-related knowledge exchange relationships. In the present article, we suggest an application of the nestedness and modularity measures in two-mode networks, in which one of the modes corresponds to people involved in software development and maintenance projects and the other mode corresponds to the projects to which these people are allocated. Such networks were studied with the purpose of answering questions related to the organizational knowledge creation and diffusion process.

Based on this context, we established the objective of identifying patterns of nestedness and compartmentalization structures in these networks by applying methods and algorithms for detecting these structures. The data for the construction of the networks were obtained from SERPRO-Serviço Federal de Processamento de Dados [Federal Data Processing Service], a public Brazilian organization which provides information and communication technology services. The study of these patterns can help to understand possible relationships between the identified structures and the knowledge creation and diffusion process 
in the studied networks. To achieve the proposed objective, it was necessary to 1) establish a theoretical framework to support the development of a methodology applicable to the structure of two-mode networks based on the empirical data obtained from the organization under study; 2) construct adjacency matrices corresponding to the allocation of people to projects in the period of 2006 to 2013 ; 3) apply nestedness and compartmentalization detection algorithms to the constructed networks and analyze and interpret the results. The study object was the systems development staff of Serpro, a Brazilian public company providing services in the area of Information and Communication Technology. From this set of people allocated to projects, a model was developed based on two-mode networks, with one of the modes being the members of software development and maintenance project teams and the second mode being the projects to which these people were allocated during projects the study period.

This article is structured in five sections. The first corresponds to this introduction. The second section develops the theoretical framework that supports the study. The third section describes the methodology used. In the fourth section, we present the application of the proposed approach and discuss the results. In the fifth and last section, we present the final considerations and the conclusion.

\section{Structure, Representation and Applications of Two-Mode Networks}

A matrix is considered two-mode if its rows and columns point to two distinct sets of entities that are related to each other as describe [6]. However, in principle, the relationships between elements of the same set are not considered. In this case, when considering social network analysis, one mode corresponds to a class of entities, called actors, nodes or vertices, whose members have ties with the members of the other class or of the second mode considered.

To exemplify the application of these structures in the context of social network analysis, [6] describe the affiliation relationships between actors and events, such as those that occur within organizations. Thus, the allocation of team members to projects is a typical example of a situation that can be modeled as a two-mode network.

Similarly, [7] classify affiliation networks as two-mode networks that aim to describe two distinct sets of actors rather than simply describing links between pairs of actors. These authors consider that because of this differentiated structure, affiliation networks require special methods of analysis. Affiliation is thus characterized as an unequivocal and unambiguous relationship between a member of one of the sets or modes of the network and the other set.

Regarding the representation of these networks, [7] Wasserman and Faust (1997) suggest some structures, among which we highlight the affiliation matrix that can be constructed so that the rows represent the actors and the columns represent the events. The intersection of a row with a column is assigned a value 
of 0 if there is no relationship between the actor and the event or with 1 if this relationship exists.

Another form of representation recommended by these authors is the bipartite graph. This representation is basically similar to the representation used in set theory, where the relationship between elements of a set is represented with the elements of the other set, using lines that indicate the connections between the elements of each set.

The decision on which type of representation should be used depends on the purpose of the analyses to be performed and the volume of elements each set contains. Thus, large volumes of data may present matrix representation problems. However, the construction of these matrices in terms of practicality and computational feasibility is superior to the representation with bipartite graphs. In summary, the type of representation to be adopted is strongly dependent on the objectives and characteristics that one desires to emphasize in relation to the represented network.

\subsection{Nestedness and Modularity in Two-Mode Networks}

In biological systems, the study of nestedness in networks refers to the property by which the interactions of a node or vertex of the network are a subset of the interactions of the next vertex with a greater degree of connections as shown in [8]. The concept of nestedness was also described in [9] and addresses the recognition of patterns in ecological communities. The concept is related to the fact that rare species predominantly inhabit richer and more diverse areas. In turn, ubiquitous species tend to inhabit both diverse and nondiverse areas.

Considering measurement, in [1] nestedness is not treated as a metric per se but rather as a concept applicable to biological metacommunities, whereby species present in poorly diverse areas represent subsets of species present in more diverse areas. Additionally, according to these authors, due to the absence of a mathematical formalism to define the concept, several distinct metrics emerged to try to quantify the concept, including the NODF (Nestedness metric based on Overlap and Decreasing Fill) algorithm described by [1]. This algorithm resulted from an improvement of that presented by [9].

For [10], modularity, compartmentalization or community formation represent distinct subsets of vertices, forming clusters or modules in which the vertices are more densely connected to each other than to other vertices. From viewpoint of the structure of two-mode networks, the two concepts provide important information, as they demonstrate the form of interaction of the network components, which can lead to the construction of hypotheses that result in new research and consequently in knowledge expansion.

\subsection{Nestedness and Modularity Patterns}

In [2] are presented several possibilities for the occurrence of bipartite network structures for biological systems. According to the authors, these structures, in 
addition to helping to establish hypotheses related to ecosystems, enable analysis from a graphical perspective that complements the mathematical modeling applied to the model.

It is also described in [2], the main patterns or models of interaction representation structures that involve nestedness and modularity. These authors present three basic forms of representing these patterns: matrix, bipartite graphs and correspondence analysis. Here, we show only the first two forms. In [2] patterns or basic structures are further identified and new structures are formed. These structures are shown in Figure 1, in a matrix representation, in which the columns represent one of the modes of the network and the rows the second mode.

Figure 1(a) shows the first model or structure, which is called gradient. In this primary model, there is a formation of groupings compartmentalized in pairs, and each element of a pair has a connection with the subsequent pair, resulting in a connection between the groupings. The second mode, shown in Figure 1 (b), corresponds to compartmentalized or modularized formation. In this formation, the modules do not have intersection points. Figure 1(c) represents the nestedness structure, and last, Figure 1(d) shows the combined structure in which there is modularity, and the formation of nestedness is observed within each module. Figure 2 shows the respective representation of each of the models shown in Figure 1, but in the form of a bipartite graph.

The visualization of these structures is obtained by the application of sorting algorithms that reorganize the matrices without, however, altering the original existing relationships. The application of the NODF (Nestedness metric based on Overlap and Decreasing Fill) algorithm described by [1] allows the resorting

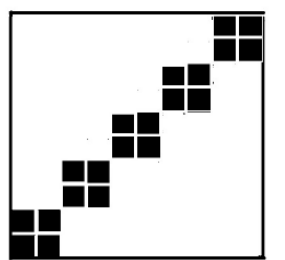

(b)

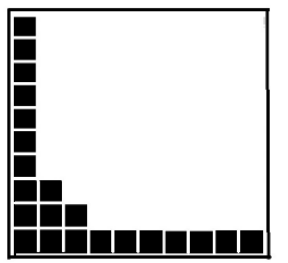

(c)

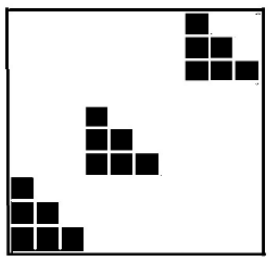

(d)

Figure 1. Matrix structure models. Source: (Authors, 2020) adapted from [2].

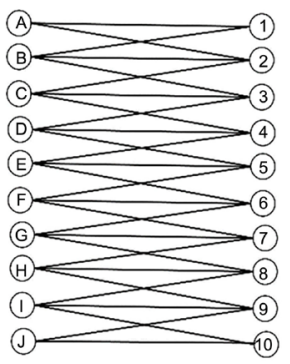

(a)

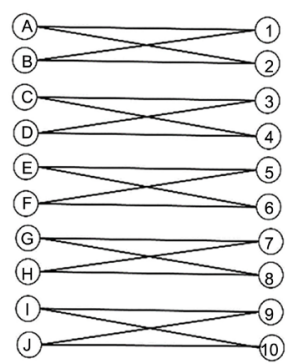

(b)

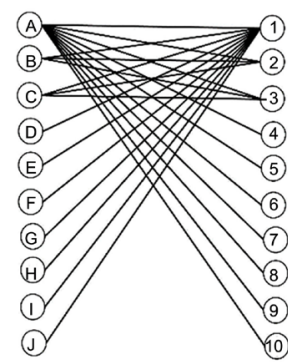

(c)

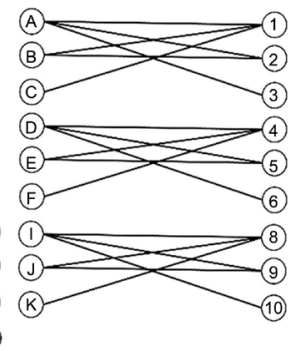

(d)

Figure 2. Matrix structure models. Source: Authors (2020) adapted from [2]. 
of the adjacency matrix in decreasing order of connections between the two modes. After the application of the algorithm, if the network structure presents nestedness patterns, these will become evident.

\subsection{Complex Two-Mode Social Networks and Applications}

In the set of modeling applications based on two-mode networks, the method of reflections, developed by [3], stands out, which made it possible to build an analysis model to map the economic development structure based on the relationships between countries and the products they export, which are mediated by skills and knowledge. In both cases, the analysis of economic systems based on network models served to understand the relationship between the capability level of countries based on categories and quantities of exported products, demonstrating the degree of diversification and ubiquity of these products. Similarly, in the present study, the support of modeling was sought to help identify network structures and patterns that facilitate knowledge creation and dissemination.

Unlike the objectives of analysis found in the studies of [9] [11] and [12] when addressing ecosystems and the objectives of Hausmann and [3] and [4], the present study sought to identify and map patterns of nestedness and modularity to better understand the structures underlying processes of organizational knowledge creation and diffusion in the network structures that are formed during the execution of projects.

\section{Materials and Methods}

The study was conducted at Serpro-Serviço Federal de Processamento de Dados [Federal Data Processing Service]. It is a public company affiliated to the Ministry of Finance that provides information and communication technology services to the public sector and is one of the largest organizations in the sector in Brazil and Latin America as shown in [13]. The departments responsible for the execution of software development and maintenance projects corresponding to the sample selected for data collection were mapped, totaling 264 people and 5077 projects. The period considered for collection was 8 years, from January 2006 to December 2013.

From the data collected, the full affiliation matrix was constructed in a Microsoft Excel spreadsheet. This matrix was transplanted into a Microsoft Access database, from which partial views of the data stratified by year were extracted, which generated other affiliation matrices. The use of the database facilitated data structuring and export in formats compatible with other software used for data treatment and analysis. Extraction was based on criteria related to the framework of the data on people allocation to software development and maintenance projects in the study period, as well as on availability and reliability. The maintenance of the record of allocation of hours by type of activity, performed daily by the employees, was essential to obtain the data. The full representation 
of the study methodological flow is shown in Figure 3.

In the present study, the NODF (Nestedness metric based on Overlap and Decreasing Fill) algorithm described by [1] was applied to the adjacency matrices that represent the affiliation of people to software development and maintenance projects and served to perform analyses analogous to those performed by [3] but focused on the study of relationships between people and projects. To apply the algorithm, the BiMat package developed by [14] was executed in MATLAB $^{\circledast}$ software.

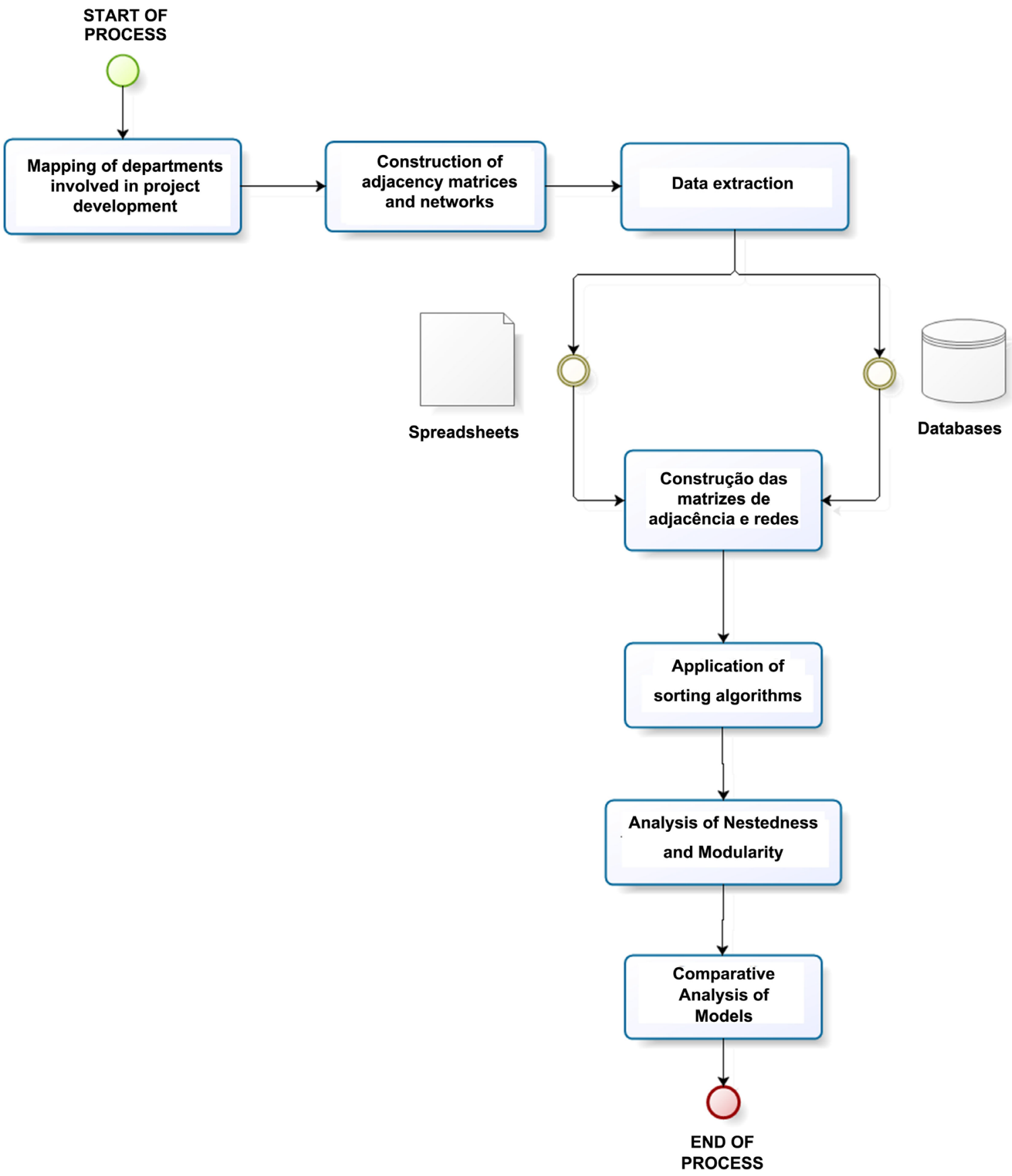

Figure 3. Flow of the research and modeling process. Source: Authors (2016). 
When [15] studied the degree of nestedness of bipartite networks of countries and their exported products, they needed to make considerations regarding the robustness of the data related to the countries' exports to establish a condition in which the country presented itself as a substantial exporter. These considerations were necessary to identify countries with a significant volume of exports made in a given period. In the case of the present study, with regard to data treatment, such considerations were not necessary because the relationship between people and projects is objectively demonstrated by the documentation present in the systems that attests to the effective performance of work on projects in the studied company, as the hours worked in a given project are performed recorded daily.

\section{Results and Discussion}

The graphical representation of the full network used for the present study is shown in Figure 4, in which we observe the arrangement of projects related to team members. To the right of the image is the network reorganized into a distribution that shows the two modes: projects, in greater numbers on the left, and people, in fewer numbers, to the right. This network represents all the relationships observed between people and projects for the entire period considered in the present study. However, this kind of visualization, not only prevents analysis that facilitates the identification of nestedness and modularization, as it is not practical to manipulate. Therefore, it is necessary to segment these relationships by year to facilitate the analyses so that we could have a view of the evolution of the nestedness and modularity formations over time.

Nevertheless, from Figure 4 we can visually perceive that the majority of nodes belong to the set of projects, which is logically acceptable, since there is a limited number of project team members, compared to the number of projects during the period of eight years considered in the study.

The analysis and interpretation of nestedness patterns are complementary to the other techniques that compose the analysis of social networks and can help
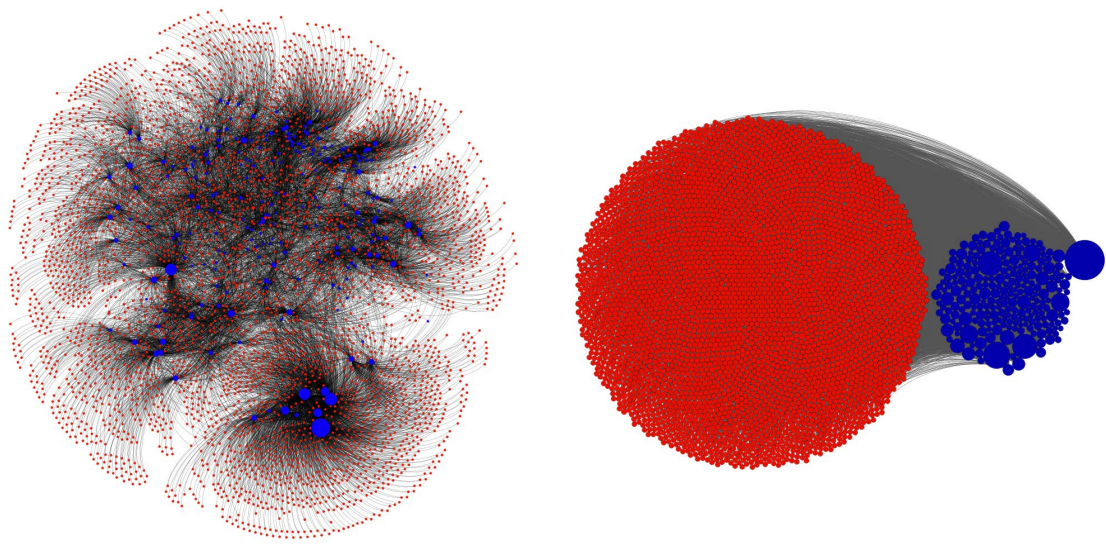

Figure 4. Complete representation of the network. Source: Authors (2020). 
understand the structures that govern the relationships between the modes considered in bipartite networks. In the case of projects and people mediated by skills and knowledge, these patterns provide a complementary analytical perspective that helps elucidate the process of knowledge diffusion in networks, starting from the individual to the collective level, to the extent that it can show the interactions between people with a more specialized knowledge profile and those with a more generalist profile, and help to predict the emergence of new capabilities that may arise from these interactions. Analyzing from viewpoint of interactions in organizational systems in the context of knowledge creation and diffusion, it can be inferred that the nestedness formations correspond to interactions between more specialized individuals and more generalist individuals. In turn, modularity refers to the formation of specialized nuclei, which may represent islands or nuclei of knowledge.

Figure 5 shows the complete matrix covering the period from 2006 to 2013, which includes the allocation of people to projects in the organization where the study was conducted. The matrix was sorted using the NODF (Nestedness metric based on Overlap and Decreasing Fill) algorithm described by [1]. The upper left extreme of a fully nested matrix represents the generalist group of species, while the group formed in the lower right quadrant represents the most unlikely combination between groups or species. In the case of the adjacency matrix of people and projects shown in Figure 5, the upper left group corresponds to the most generalist nucleus of people participation in projects. In other words, it represents the most likely relationships between people and projects that correspond to the maximum level of ubiquity. Conversely, in the direction of the opposite diagonal, the most unlikely participations of people in projects are represented. The occurrences present below the isocline represent the unexpected relationships, while those above the line represent the expected relationships. According to [2], the detection of interaction structures in matrix representations can be obtained by rearranging rows and columns since their permutation does not alter the original structure of the data. This reordering allows visual identification and simultaneously evaluating the degree of nestedness and the formation of grouping modules or nuclei in the matrix.

The temporal variations of the relationships that may influence the nestedness patterns can be partially explained by the dynamics of knowledge, in that the exchange of knowledge between members of project teams allows the socialization of knowledge in the sense described by [16], allowing collaboration and knowledge sharing. Consequently, these patterns may undergo transformations. In this case, a dynamic representation model based on agent modeling can portray this change in a clearer and more objective way. Such temporal variations

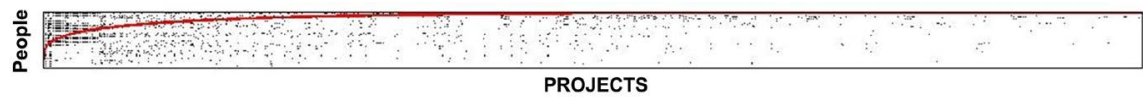

Figure 5. Complete matrix ordered by the NODF algorithm. Source: Authors (2020). 
are demonstrated in [17], who studied other network metrics and their influences on the knowledge creation and diffusion process in a longitudinal study.

The presentation of structures with a high degree of nestedness in the matrices obtained from the people-project affiliation networks studied shows coherence with what [18] discusses when studying biological systems. Similar to what the aforementioned author presents, knowledge networks also seem to have an inverse relationship between the degree of connectivity (nestedness) and modularity. This may indicate that nestedness favors knowledge diffusion and minimizes the effects of compartmentalization or modularity that forms knowledge nuclei. Figure 6 shows two matrices that represent the relationships between people and projects for the year 2009. The representation of Figure 6(a) refers to the relationship before the sorting applied by the algorithm of [1] implemented in the BiMat package, and the matrix shown in Figure 6(b) represents the sorted matrix after the application of the algorithm.

The graph in Figure 7 shows the evolution of nestedness between 2006 and 2013. The filled points represent the measured nestedness values, while the hollow points represent the random expectations. In the observed period, with the exception of 2006, in which the information about allocations to projects was in an initial stage, high nestedness values are observed in most of the years.

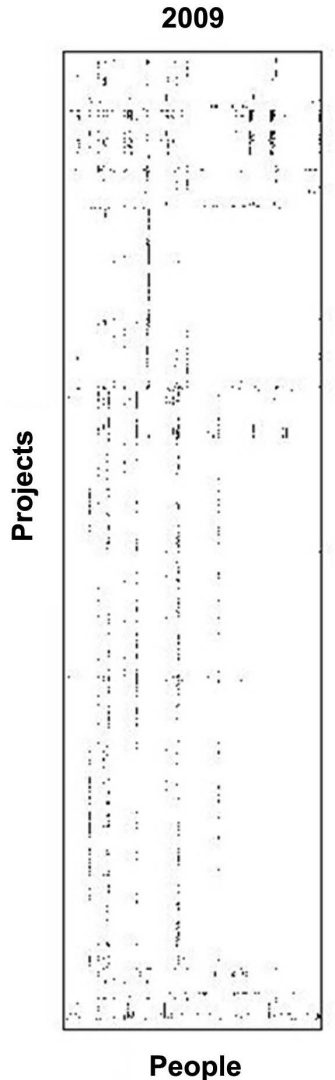

(a)

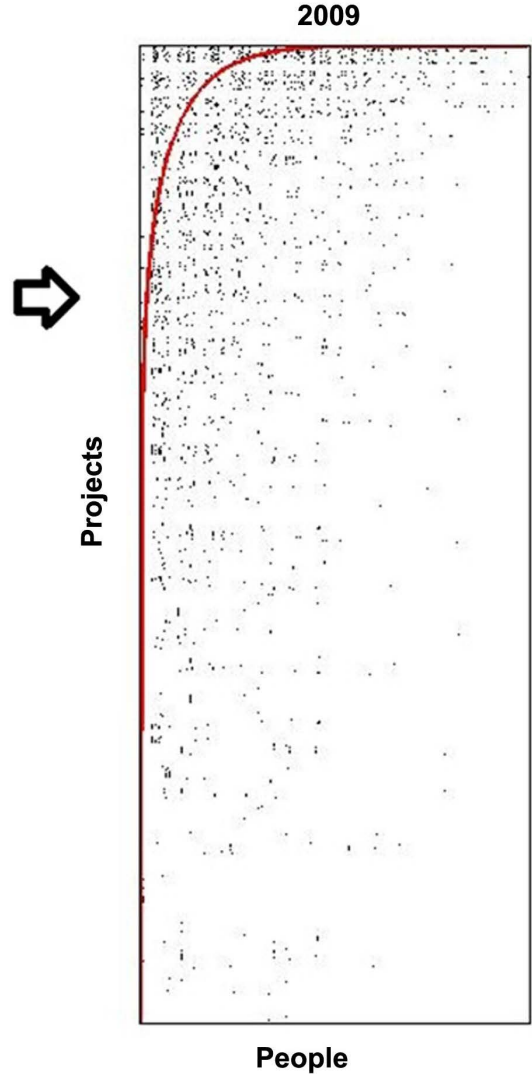

(b)

Figure 6. 2009 matrix before and after the application of the NODF. Source: Authors (2020). 


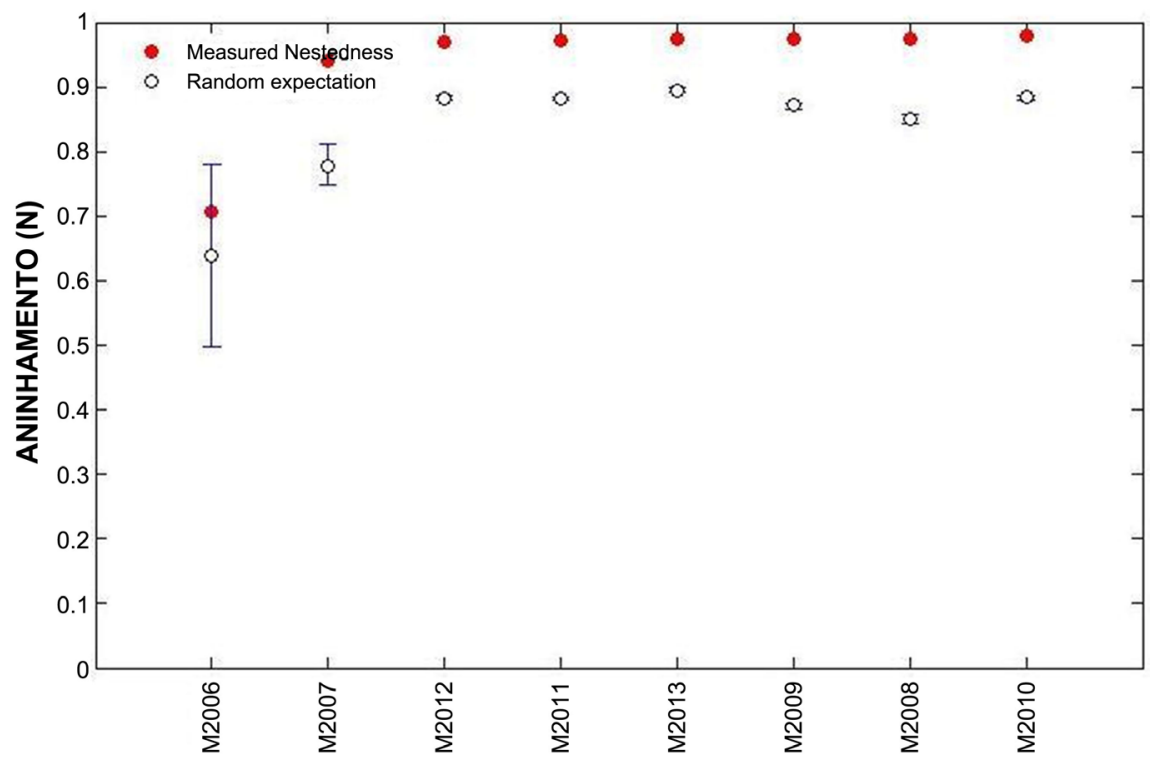

Figure 7. Evolution of nestedness between 2006 and 2013. Source: Authors (2020).

The detection of nestedness can be observed graphically in Figure 8 for 2007. The figure shows a structure similar to those exemplified by [14] and represented in Figure 2(c), which reinforces the expectation of nestedness demonstrated by the results found for the studied network.

Figure 8 shows the relationship between people and projects in 2007 in the form of a bipartite graph. This relationship is indicated by the lines that connect the elements of the two sets. Figure 9 shows the same visualization pattern for the networks built between 2006 and 2013.

Table 1 shows in column $\mathbf{N}$ the nestedness values for each of the years considered in the study. Again, with the exception of 2006, for which the data were still insufficient to represent the work performed, each year presented nestedness values very close to 1 .

In the study period, with the exception of 2006, the nestedness structure can be clearly identified. The justification for the differentiated structure in the first year considered in the study is that in that year, data collection was started using the system for allocating people to projects and only a few pilot projects were included in the control system. In Figure 10, we show the modularity structures for the same year. These structures are shown in the figure by the rectangular formations that appear in varying sizes. Within the larger modules, it is possible to observe the presence of nestedness structures, which correspond to the triangular formation in the representation of the relationships between people and projects. This formation indicates that for that year, a mixed or combined configuration of nestedness and modularity can be observed, compatible with the pattern represented in Figure 1(d).

For [2], modularity or compartmentalization in the context of ecological communities represents a pattern of interactions that form a recognizable subset of relationships between the two modes of the network. Similarly, in the case of 


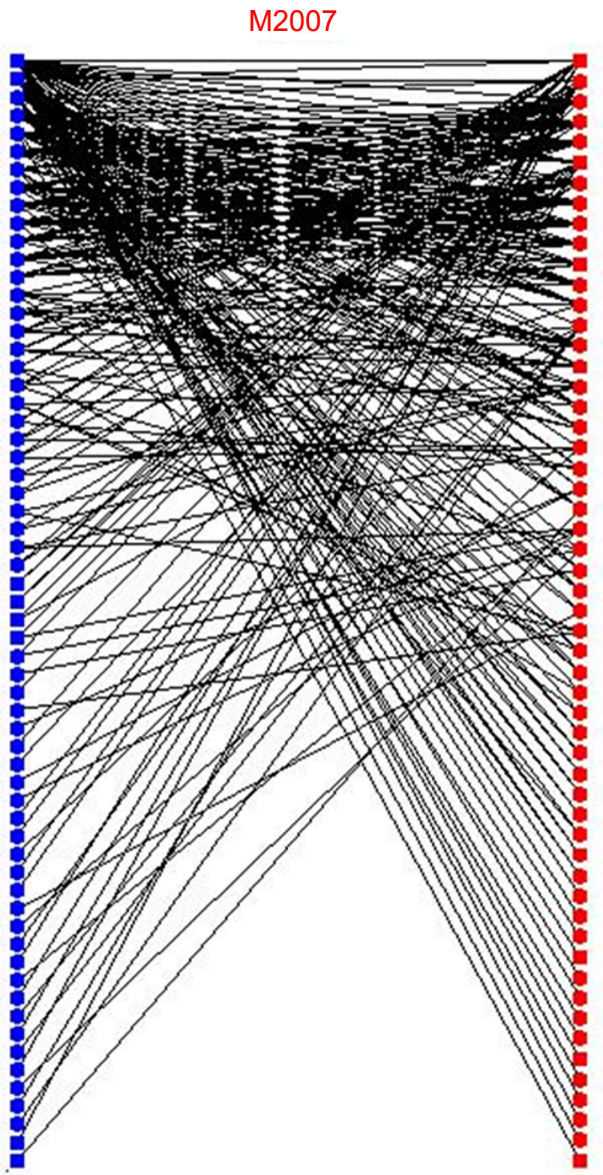

Figure 8. Bipartite representation of the network in 2007. Source: Authors (2020).

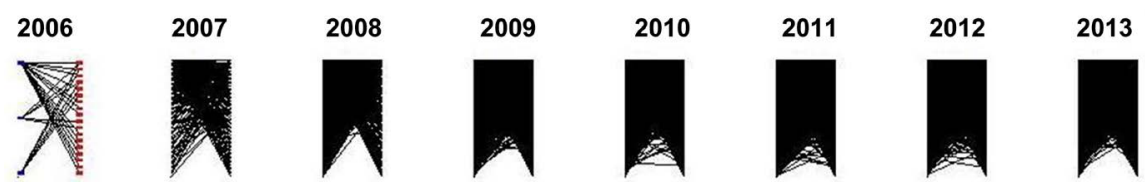

Figure 9. Representation of the nestedness of the networks from 2006 to 2013. Source: Authors (2020).

Table 1. Representation of the nestedness of the networks from 2006 to 2013.

\begin{tabular}{ccccc}
\hline Year & $\mathbf{N}$ & $\mathbf{N}$ mean & $\mathbf{N ~ z - s c o r e}$ & $\mathbf{N}(\%)$ \\
\hline 2006 & 0.70786 & 0.6388 & 0.92508 & 83 \\
2007 & 0.93935 & 0.77681 & 9.9461 & 100 \\
2008 & 0.97504 & 0.85018 & 32.3538 & 100 \\
2009 & 0.97416 & 0.87122 & 46.7369 & 100 \\
2010 & 0.98042 & 0.88464 & 53.5509 & 100 \\
2011 & 0.97216 & 0.88152 & 51.4986 & 100 \\
2012 & 0.96885 & 0.88319 & 53.457 & 100 \\
2013 & 0.97329 & 0.89418 & 44.7661 & 100 \\
\hline
\end{tabular}

Source: Authors (2020). 


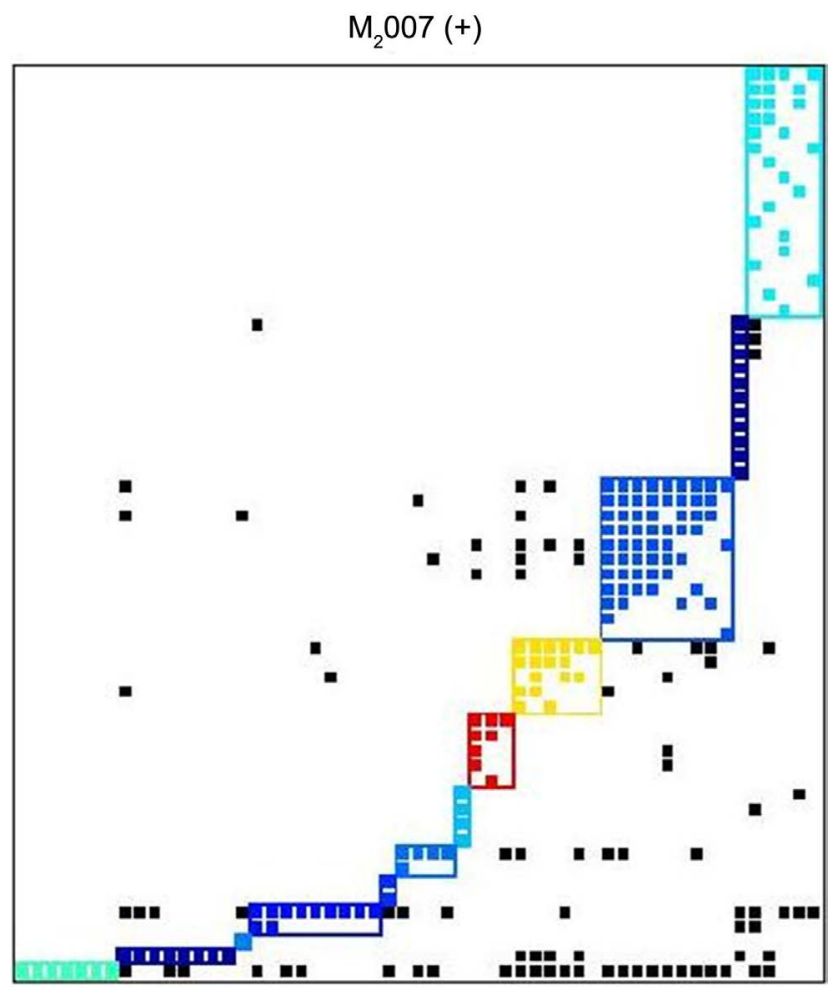

Figure 10. Modularity structure in 2007. Source: Authors (2020).

the present study, modularity represents a subset of relationships between people and projects, characterizing groupings aimed at carrying out projects. These groupings must also be associated with a set of specific knowledge that will support the performance of tasks.

Modularity corresponds to the formation of clusters, which in the case of the study of knowledge networks, represent small nuclei of specialized knowledge or the so-called "islands of knowledge". From the point of view of the process of knowledge creation and diffusion considering software development teams, such structures represent at the same time highly specialized groups, but also the possibility of knowledge isolation within those groups. In contrast the measure of nestedness identified as a combined pattern in those groups, represents the possibility integrating specialized knowledge with generalist knowledge. Such integration is useful and promotes knowledge exchange between the two categories.

The graph in Figure 11 shows the modularity values of the people represented in project matrices obtained between 2006 and 2013. The measured modularity corresponds to the filled points, while the random expectation corresponds to the hollow points.

As both modularity and nestedness can be identified in seven of the eight years studied, we can infer that the environment provided by the organization studied allows a balanced process of knowledge exchange and favours knowledge creation and diffusion between groups formed by software development team members.

The graph in Figure 12 shows the modularity values for 2006 to 2013 while 

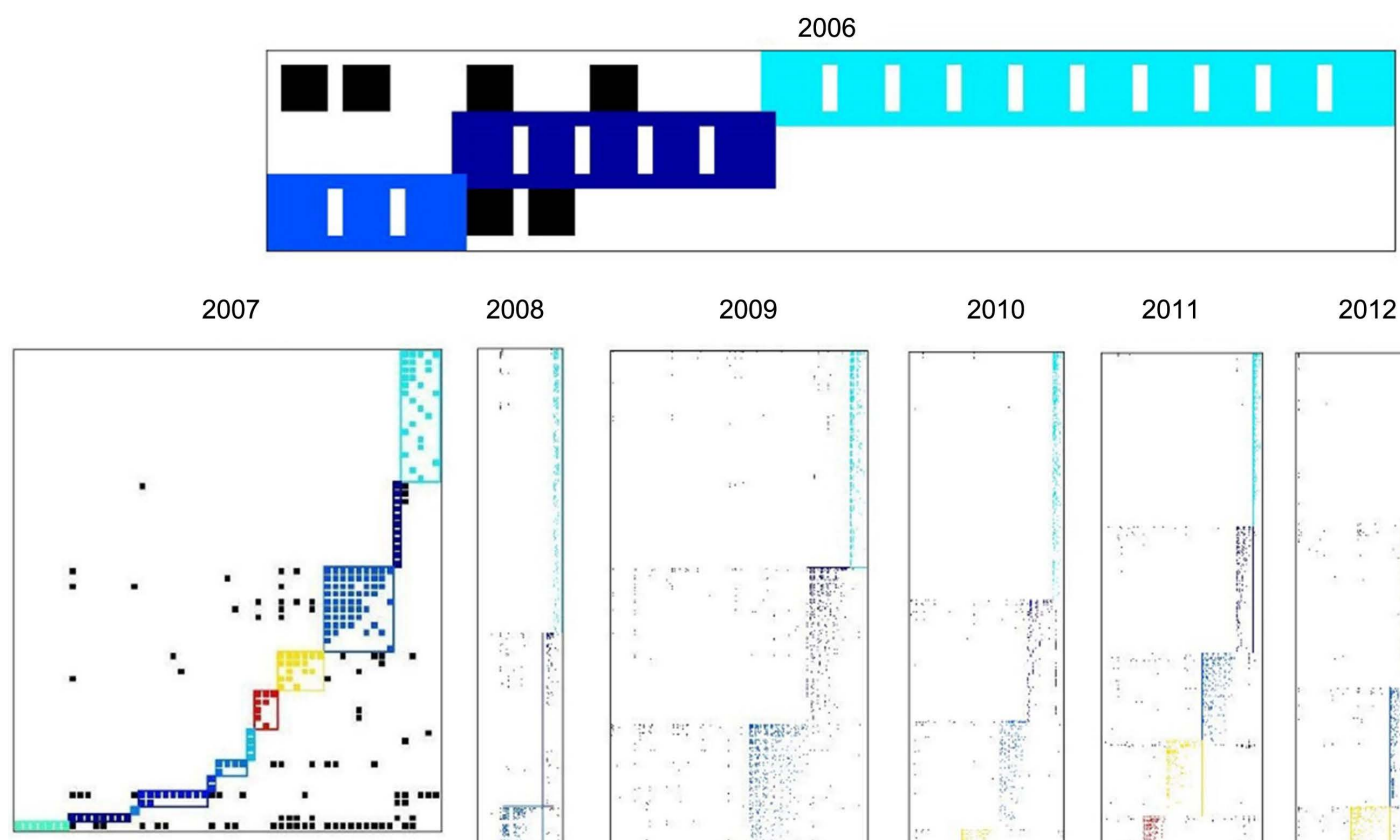

2008

2009
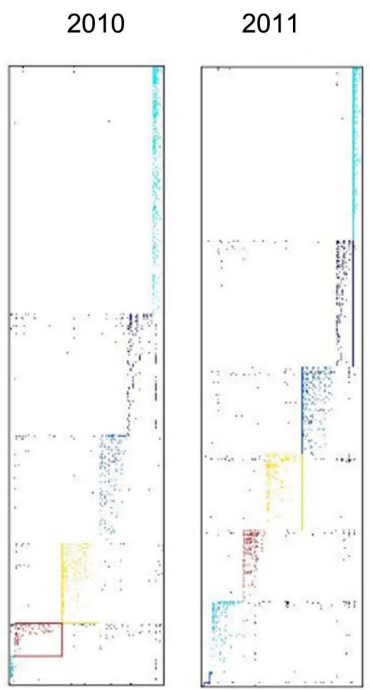

2012

2013
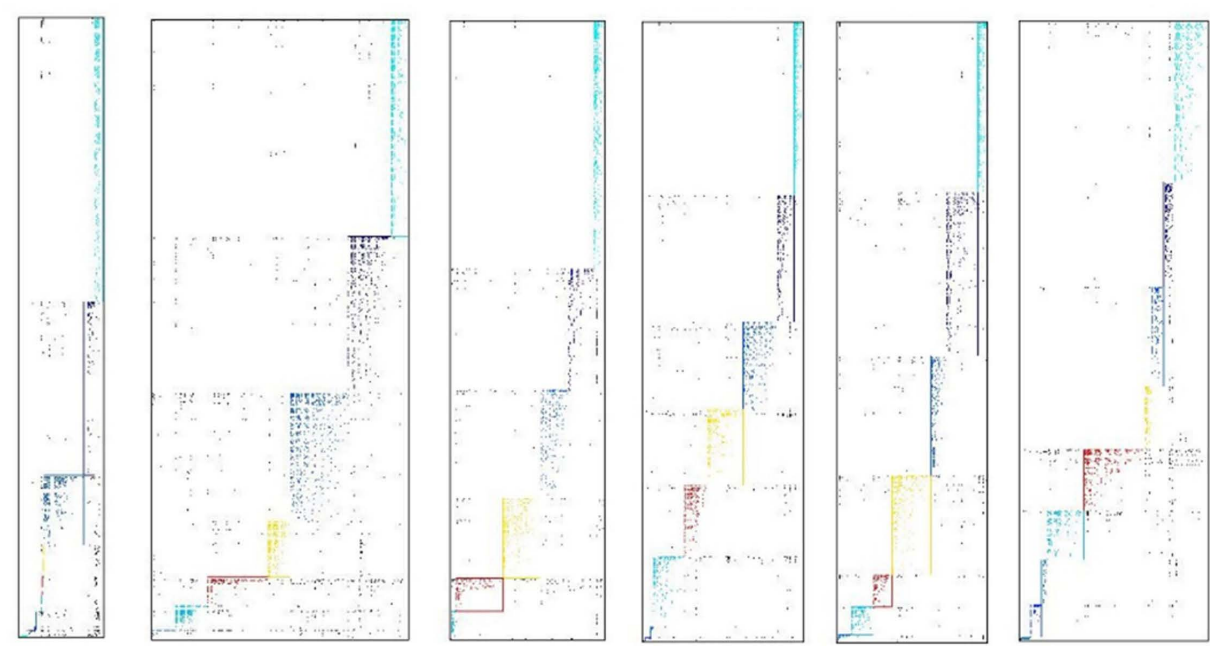

Figure 11. Representation of the modularity of the networks from 2006 to 2013. Source: Authors (2020).

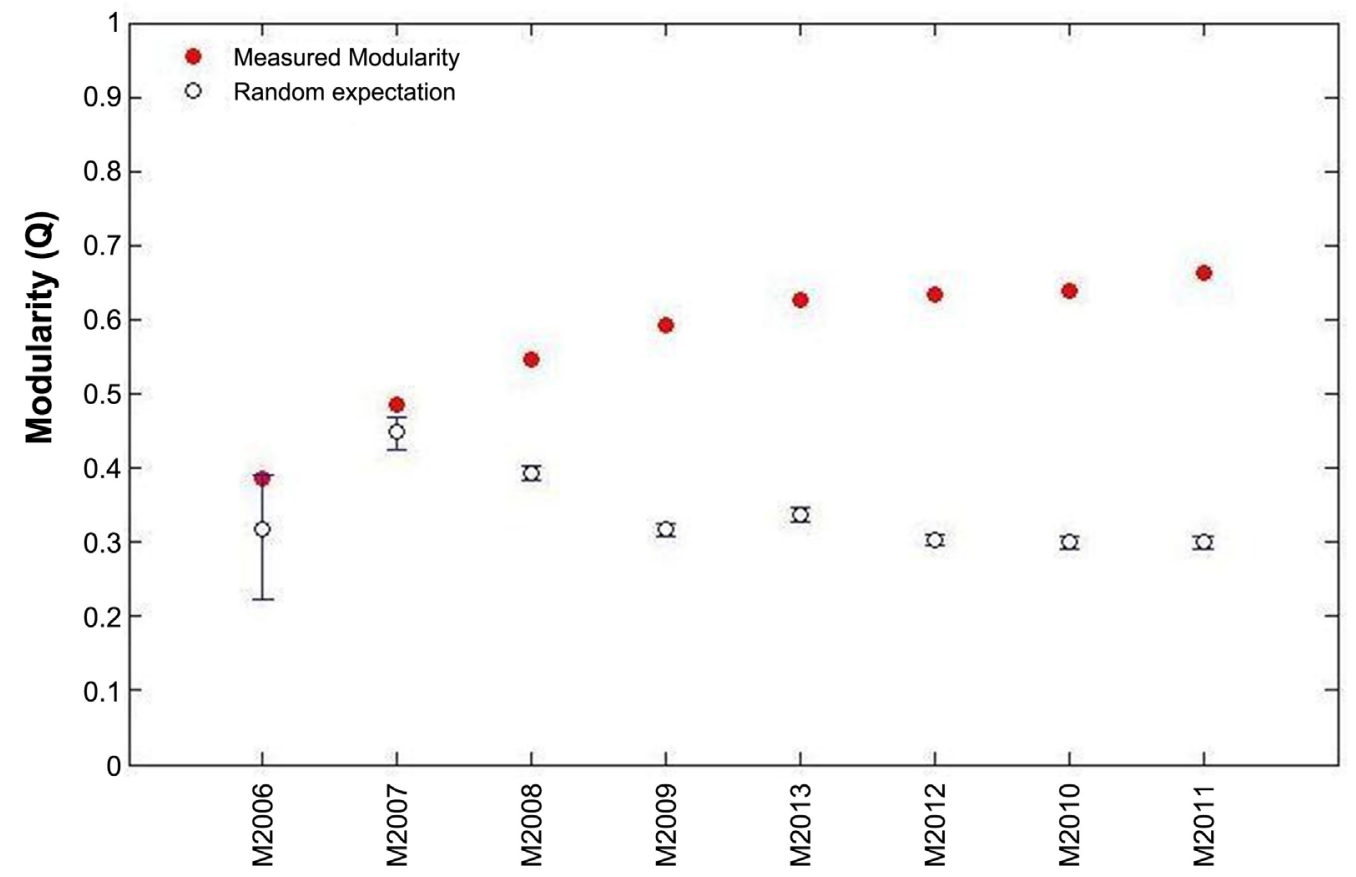

Figure 12. Evolution of modularity between 2006 and 2013. Source: Authors (2020).

Figure 10 highlights the formation of modular structures in 2007. Table 2 presents some of the statistics calculated for the networks obtained in the Gephi software. It highlights the modularity values that represent the formation of nuclei in the context of knowledge creation and diffusion. 
Table 2. Evolution of modularity between 2006 and 2013.

\begin{tabular}{ccccccc}
\hline Year & Nodes & Edges & Diameter & Modularity & $\begin{array}{c}\text { No. of } \\
\text { communities }\end{array}$ & $\begin{array}{c}\text { Average Clustering } \\
\text { Coefficient }\end{array}$ \\
\hline 2006 & 21 & 27 & 3 & 0.201 & 3 & 0.697 \\
2007 & 117 & 683 & 8 & 0.370 & 4 & 0.860 \\
2008 & 745 & 1849 & 8 & 0.540 & 8 & 0.003 \\
2009 & 735 & 35,880 & 4 & 0.526 & 4 & 0.785 \\
2010 & 1105 & 98,687 & 4 & 0.463 & 5 & 0.785 \\
2011 & 1074 & 13,592 & 4 & 0.451 & 7 & 0.901 \\
2012 & 1080 & 4512 & 7 & 0.689 & 12 & 0.813 \\
2013 & 859 & 36,618 & 4 & 0.580 & 7 & 0.796 \\
\hline
\end{tabular}

Source: Authors (2020).

\section{Conclusions}

This study aimed to identify patterns of nestedness and modularity in affiliation networks formed by the participation of people in software development and maintenance projects from 2006 to 2013. Our approach sought to relate the parts and structures of nestedness and modularity identified in the studied networks with issues associated with knowledge creation and diffusion in the context of system development and maintenance project teams. Such structures can help to establish hypotheses about the knowledge exchange process in the organizational environment.

The historical data referring to the allocation of team members to projects enabled the construction of the affiliation network and the application of the algorithm for the detection of nestedness and modularity structures in the networks. The basic statistics that allow the interpretation and analysis of the network are obtained, respecting its structure, considering that two-mode networks have peculiarities that alter the form of calculation and the interpretations for some metrics applicable to one-mode networks, and cause other metrics to cease to make sense or require a different interpretation. The applied method enabled the construction of the networks, preparing them for the analysis of the relationships based on the historical data regarding the allocation of team members to projects. In addition, the study demonstrated that it was possible to apply the method to other affiliation networks by aggregating data and characteristics specific to such networks.

The application of the method was made possible by obtaining data on the allocation of people to projects from the allocation of worked hours recording system used in the organization. In addition, the use of multiple tools such as spreadsheets, databases and algorithms developed for the detection of nestedness and modularity structures helped to build a methodological path to obtain results. This study contributes to expanding the knowledge about the application of the metrics addressed, demonstrating new possible applications different from 
those performed in biological systems.

The networks analyzed showed a high nestedness pattern in the period between 2007 and 2013. Modularity patterns were also identified during the analyzed period but to a lower degree. From the viewpoint of the knowledge creation and diffusion process, these results suggest high cohesion of the studied networks, which may demonstrate the robustness of these networks in relation to knowledge diffusion. The evidence for this consideration comes from the fact that nestedness also represents a high level of network connection. These results are consistent with the modularity indices found, which, because they are lower, suggest that the formation of nuclei or "islands" of knowledge occur, albeit to a lower degree. In addition, considering the exchange of knowledge between members of more specialized teams with members of less specialized teams facilitates knowledge exchange and diversification. In other words, the diffusion of knowledge seems to be facilitated by the interactions between groups of more specialized people with groups of less specialized people. In turn, the diversity of projects can also favor knowledge diffusion, given the diversification of areas of knowledge involved in projects of different origins. The modularity found can also be explained by the business areas that involve sectorized knowledge.

This study did not address the categorization of knowledge considered as an attribute of the relationships between people and projects in the affiliation matrices. Aggregating this knowledge can help to better understand the distribution of this knowledge in networks.

As an extension of the present study, a more in-depth analysis of the indices found is proposed, comparing them with other indices related to network cohesion, such as the average degree of the one-mode projection network derived from the original two-mode network. Also, the average degree of clustering could be obtained in the same way. Another possibility of extending this work is the inclusion of attributes that characterize and qualify the knowledge used in the interaction between team members and projects. This characterization can help to better understand the quality of relationships in terms of knowledge creation and diffusion, which can reinforce the argument about the relationship between knowledge and the modularity and nestedness metrics.

However, it would be possible to establish a generic structure, in the form of a data model, that could absorb data from different affiliation networks, also enabling the aggregation of additional characteristics, attributes or parameters, to characterize or categorize the network nodes, thus enabling the expansion of the model and enrichment of the analyses. This could be a way to minimize the effects of the particularities of bipartite network relationships. The construction of this model, together with the construction of the software to support the calculations, constitutes the recommendations for future studies.

\section{Acknowledgements}

The authors thank the Federal Data Processing Service-Serpro for allowing to 
use the research data, as well as the support given to the whole process of the research.

\section{Conflicts of Interest}

The authors declare no conflicts of interest regarding the publication of this paper.

\section{References}

[1] Almeida-Neto, M., et al. (2008) A Consistent Metric for Nestedness Analysis in ecological Systems: Reconciling Concept and Measurement. OIKOS, 117, 1227-1239. https://doi.org/10.1111/j.0030-1299.2008.16644.x

[2] Lewinsohn, T.M., Loyola, R.D. and Prado, P.I. (2006) Matrizes, redes e ordenações: a detecção de estrutura em comunidades interativas. Oecologia Brasiliensis, 10, 90-104. https://doi.org/10.4257/oeco.2006.1001.06

[3] Hidalgo, C A and Hausmann, R. (2009) The Building Blocks of Economic Complexity. Proceedings of the National Academy of Sciences of the United States of America, Cambridge, 106, 10570-10575.

https://doi.org/10.1073/pnas.0900943106

[4] Pietronero, L., Cristelli, M. and Tacchella, A. (2013) New Metrics for Economic Complexity: Measuring the Intangible Growth Potential of Countries. .

[5] Bastos, V.B., and Santos, M.V. (2007) Redes sociais informais e compartilhamento de significados sobre mudança organizacional. Revista de Administração de Empresas, 47, 27-39. https://doi.org/10.1590/S0034-75902007000300003

[6] Borgatti, S.P. and Halgin, D. (2003) Analyzing Affiliation Networks.

[7] Wasserman, S. and Faust, K. (1994) Social Network Analysis. Cambridge University Press, New York. https://doi.org/10.1017/CBO9780511815478

[8] Bascompte, J., et al. (2003) The Nested Assembly of Plant-Animal Mutualistic Networks. (2003) Proceedings of the National Academy of Sciences of the United States of America, 100, 9383-9387. https://doi.org/10.1073/pnas.1633576100

[9] Patterson, B.D. and Atmar, W. (1986) Nested Subsets and the Structure of Insular Mammalian Faunas and Archipelagos. American Heart Journal, 90, 795-803.

[10] Danon, L., Albert, D. and Duch, J. (2005) Comparing Community Structure Identification. Journal of Statistical Mechanics. Theory and Experiment, 2005, P09008. https://doi.org/10.1088/1742-5468/2005/09/P09008

[11] Almeida-Neto, M., Guimarães Jr., P.R. and Lewinsohn, T.M (2007) On Nestedness Analyses: Rethinking Matrix Temperature and Anti-Nestedness. Oikos, 116, 716-722. https://doi.org/10.1111/j.0030-1299.2007.15803.x

[12] Ulrich, W. (2009) Nestedness Analysis as a Tool to Identify Ecological Gradients. Ecological Questions, 11, 27-34. https://doi.org/10.12775/v10090-009-0015-y

[13] Serpro, S.F., De, P. and De, D. (2016) Site Institucional. www.serpro.gov.br

[14] Flores, C.O., Poisot, T., Valverde, S. and Weitz, J.S. (2016) BiMat: A MATLAB Package to Facilitate the Analysis of Bipartite Networks. Methods in Ecology and Evolution, 7, 127-132. https://doi.org/10.1111/2041-210X.12458

[15] Bustos, S., et al. (2012) The Dynamics of Nestedness Predicts the Evolution of Industrial Ecosystems. PLoS ONE, 7, e49393. 
https://doi.org/10.1371/journal.pone.0049393

[16] Nonaka, I. and Takeuchi, H. (1997) Criação de conhecimento na empresa: como as empresas japonesas geram a dinâmica da inovação. Rio de Janeiro.

[17] Dos Santos, J.L. and Sampaio, R.R. (2019) Temporal Analysis of the Diffusion of Knowledge in Networks of Software Maintenance and Development Project Team. Social Networking, 8, 122-146. https://doi.org/10.4236/sn.2019.83009

[18] Pires, M.M., et al. (2011) The Nested Assembly of Individual-Resource Networks. Journal of Animal Ecology, 80, 896-903.

https://doi.org/10.1111/j.1365-2656.2011.01818.x 